\title{
What is the evidence on cholinergic medication for tardive dyskinesia?
}

\author{
Vishal Bhavsar
}

COMMENTARY ON... COCHRANE CORNER ${ }^{\dagger}$

\begin{abstract}
SUMMARY
Antipsychotic drugs are associated with movement disorders, especially with long-term use. Tardive dyskinesia, a condition characterised by repetitive involuntary muscle activity, is considered to be the most chronic, distressing and disabling of antipsychotic-associated movement disorders. There is theoretical justification for the use of cholinergic drugs in tardive dyskinesia, and they are used in clinical practice. A Cochrane systematic review synthesised randomised controlled trial data evaluating the effectiveness of cholinergic drugs for tardive dyskinesia, and for a range of secondary outcomes, including quality of life. In line with the authors of the review, this Commentary concludes that much higherquality evidence on the use of cholinergic drugs in tardive dyskinesia is necessary, and that a patient with tardive dyskinesia should be offered the opportunity to try a newer cholinergic drug, ideally in the context of a well-conducted and reported clinical trial. At the same time, given uncertainty regarding clinical effectiveness, and in view of their accepted adverse effects, it would be understandable if a person with tardive dyskinesia decided to avoid cholinergic drugs.
\end{abstract}

\section{DECLARATION OF INTEREST}

None.

\section{KEYWORDS}

Antipsychotics; tardive dyskinesia; cholinergic drug.

Psychosis is a serious mental illness affecting around $1 \%$ of the general population and is manifest in fixed odd beliefs (delusions), unusual perceptions (hallucinations), and disordered thinking and communication (thought disorder). Antipsychotic-induced tardive dyskinesia is a common problem in mental health services, typically affecting patients taking typical antipsychotics over long periods of time (Kane 1982). Antipsychotics are the mainstay of medical treatment for symptoms of psychosis and are widely used as maintenance therapy in those who have experienced previous psychotic episodes.
Historically, maintenance therapy with antipsychotic drugs for longer than 2 years after a psychotic episode has been the norm, rather than the exception.

Antipsychotics are associated with a range of adverse unintended effects, including movement disorders. Tardive dyskinesia, a condition characterised by repetitive, involuntary muscle activity, is the most chronic, distressing and disabling of these. Patients with tardive dyskinesia usually present with repetitive tongue protrusion, lateral or rotational movements of the jaw, eye blinking, and/or smacking, puckering or pursing of the lips. Onset is usually gradual, and the condition is regarded as enduring (Gelder 1989).

Although tardive dyskinesia is associated with treatment with antipsychotic drugs, the relationship with dose is unclear: dose increases can result in temporarily reduced symptoms, and withdrawal of antipsychotics can be associated with exacerbation of symptoms. The prognosis for tardive dyskinesia appears to be influenced by the degree/severity of the underlying psychiatric illness, the duration of antipsychotic therapy and the age of the patient. More than $20 \%$ of patients treated with older first-generation antipsychotics for longer than 3 months develop tardive dyskinesia. Around 5\% of working-age adults taking antipsychotics will develop tardive dyskinesia over the course of a year; that figure is around 30\% in elderly people. Older patients are more likely to develop tardive dyskinesia and are less likely to display spontaneous remission (Tammenmaa-Aho 2018).

The mechanism by which antipsychotics increase the risk of tardive dyskinesia is unknown. Longterm blockade of dopamine receptors could result in imbalance in the activity of striatal dopaminergic and cholinergic neurons (Gerlach 1988). Alternatively, tardive dyskinesia has also been hypothesised to result from the elaboration of cytotoxic free radicals and resulting cortical neuronal toxicity (Lohr 2003). It is probably incorrect to describe tardive dyskinesia as a disappearing problem - in fact, its occurrence with second-generation antipsychotics may be similar to that with the older agents
ROUND THE CORNER
Vishal Bhavsar is a general adult psychiatrist with South London and Maudsley NHS Foundation Trust, and post-doctoral researcher at the Institute of Psychiatry, Psychology and Neuroscience, King's College, London, UK.

Correspondence Dr Vishal Bhavsar, Department of Psychosis Studies, King's College London, Institute of Psychiatry, Psychology and Neuroscience, Denmark Hill, London SE5 8AZ, UK. Email: vishal.2.bhavsar@ kcl.ac.uk

\section{Copyright and usage}

(C) The Royal College of Psychiatrists 2018

${ }^{\dagger}$ See p. 288, this issue. 
(Stegmayer 2018). Increased off-label prescribing, an ageing population and continued use of first-generation drugs in poorer countries mean that tardive dyskinesia remains an important challenge in the management of patients with psychosis. The CATIE trial of antipsychotic drugs followed patients for about 4 years, finding that 17\% developed tardive dyskinesia (Miller 2005). On the basis of the hypothesis that tardive dyskinesia results from damage to cholinergic cells in the striatum, procholinergic medications have been used to treat it.

In the review featured in this month's Cochrane Corner, Tammenmaa-Aho et al (2018) evaluated cholinergic medications for tardive dyskinesia. These included older cholinergics which increased synthesis, and therefore circulating levels, of acetylcholine, thereby allowing for increased/enhanced cholinergic transmission in the midbrain. They also evaluated drugs that have been tested because their effects on the body are similar to those of acetylcholine, such as deanol and meclofenoxate. Newer cholinergic compounds, such as galantamine and rivastigmine, have emerged for the treatment of Alzheimer's disease, and these drugs were also included in the review, because of their mechanism of action and their potential for use for treating tardive dyskinesia.

Since the publication of good-quality randomised trials - CATIE (McEvoy 2006) and CUTLASS (Jones 2006) - showing broadly equivalent effectiveness of all antipsychotic drugs for the symptoms of psychosis/schizophrenia, tolerability has emerged as a key consideration in prescribing for psychosis. In this regard, the possibility that newer, atypical antipsychotics might offer lower risk of tardive dyskinesia has been attractive for prescribers, but this has not been borne out by research evidence. Therefore, tardive dyskinesia remains a chronic, irreversible, disabling and largely unexplained side-effect related to all known antipsychotic drugs, and it is associated with poorer quality of life and reduced life expectancy.

\section{The Cochrane review}

Tammenmaa-Aho et al (2018) aimed to determine the effectiveness of cholinergic drugs for treating antipsychotic-induced tardive dyskinesia in people with psychotic disorders and other serious mental illness. Secondarily, the review authors were interested in any relation between effectiveness of cholinergic mediation and duration of cholinergic treatment, and in assessing any difference in effectiveness between the different types of cholinergic drug. Finally, the investigators examined whether response of tardive dyskinesia to cholinergic treatment was affected by the patients' age, in particular because the frequency of tardive dyskinesia is thought to be higher in those who are older. The review is part of a series of Cochrane reviews on this topic that began in 1997 (Soares-Weiser 1997), with an intervening update in 2002 (Tammenmaa 2002). Risk factors for the development of tardive dyskinesia are discussed in a recent review by Solmi et al (2018).

The authors included all randomised controlled trials addressing the review question. Quasi-randomisation, for example, by assigning participants to the treatment/control intervention on the basis of the day of the week of their enrolment, was also allowed. Studies were included if the population recruited into the trials were people with schizophrenia or any other serious mental illness. No limits were placed on the type of diagnostic criteria used to determine the presence of schizophrenia, and neither were limits placed based on gender, age or nationality. For inclusion in the systematic review, studies needed to have randomised individuals who had required a stable dose of antipsychotic treatment for a period longer than 3 months and who had developed tardive dyskinesia during antipsychotic treatment. Tardive dyskinesia could be identified by any means, and at any time during the trial, as long as it had been diagnosed on more than one occasion (for example, at baseline and on one more occasion). The review assessed a range of cholinergic compounds as interventions of interest. Allowable control groups were those treated with placebo or with no treatment. This particular update included studies comparing cholinergic drugs against any other tardive dyskinesia treatment.

\section{Outcomes of interest}

The primary outcomes for the included studies were clinical improvement in tardive dyskinesia (defined as improvement of greater than $50 \%$ on any tardive dyskinesia scale) and the absence of adverse effects of the treatment (cholinergic drugs), in the form of clinically significant adverse effects. The reviewers were also interested in any tardive dyskinesia improvement (even if it did not meet the $50 \%$ threshold defined as the primary outcome), differences in any continuous measures of quality of life, and differences in any continuous measures of functioning. Other secondary outcomes gathered by the reviewers on the returned studies included assessments of changes in general mental state, differences in acceptability and adverse effects of the treatment (including need for antiParkinsonian drugs), hospital admissions, economic outcomes, social network and quality-of-life measures, behavioural measures (such as agitation and aggression) and cognitive changes. 


\section{Search method, inclusion criteria and data extraction}

The search for studies was carried out by electronic searching of the Cochrane Schizophrenia Group's Study-Based Register of Trials. This contains an updated list of trials compiled by searches of major electronic resources (AMED, BIOSIS, CINAHL, Embase, MEDLINE, PsycINFO, PubMed, and registries of clinical trials). In addition, the reviewers looked at reference lists and contacted authors for results of unpublished trials. Having identified candidate studies for inclusion, the abstracts of these studies were screened by two of the investigators. Disagreements on whether a study should beincluded were resolved by discussion between the raters.

Two raters then extracted data from the included studies using simple forms. Where results of an included study pertained to continuous measures (e.g. symptoms), for inclusion the psychometric properties of the instrument needed to have been reported in the literature previously and to be used in an unmodified form in the particular included study. The reviewers also aimed to gather information on whether the measures were self-reported or collected by independent raters. Change in outcome data for individual participants, calculated by the difference in outcome between baseline and some subsequent measurement, was preferred for inclusion, but endpoint data were included if change data were not available. In the analysis, endpoint and change data was combined. Outcome measures were harmonised between studies to aid comparison, where possible. Continuous measures were then converted to dichotomised variables, indicating response versus non-response. Bias in included studies (Box 1) was assessed by consensus based on criteria in the Cochrane Handbook for Systematic Reviews (Higgins 2011).

Cluster randomised trials were included, and statistical handling was guided by advice. Cross-over trials were included, but only data from the first phase, to avoid carry-over effects affecting the second phase. Trials with multiple treatment groups, relevant to the review topic, were allowed.

Studies with greater than 50\% missing data on outcomes were excluded. Missing data on continuous measures were imputed on the basis of other data included in the review. Heterogeneity among the included studies was assessed by addressing each study in turn in terms of the clinical populations studied and the methods employed. Statistical heterogeneity in estimates of effect was evaluated by graphical inspection of forest plots and the $I^{2}$ statistic. Funnel plots were not used to assess publication bias, as the number of studies was too small. To provide a quantitative summary
BOX 1 Addressing sources of bias in systematic reviews

\section{Pre-specified design}

The absence of a pre-specified review protocol introduces the risk that inclusion criteria and other important decisions have been made ad hoc and may therefore reflect the prior expectations of the researchers.

Duplicate study selection and extraction

The presence of two independent raters reduces the possibility of human errors in the selection of studies for inclusion and in the extraction of data.

\section{Comprehensive literature search}

As many appropriate databases as possible should be searched, in order that the search is comprehensive. Searches should be accompanied by approaches to systematic reviewers, reviewing references of included studies, and consulting reviews and textbooks. Search terms should be reported and some statement made about how the terms were derived, for example in collaboration with librarians and literature search experts. The reviewers should state whether publication status was an exclusion criterion, e.g. did they exclude grey literature, or papers not written in English?

A list of excluded and included studies

This should be provided
Characteristics of included studies reported

Information on participants, exposures, outcomes, analyses and results of included studies should be reported.

Assessment of scientific quality of included studies

Any pre-specified criteria for excluding particular types of study, e.g. non-randomised studies, should be stated. Scientific quality of included studies should be assessed and reported, and used appropriately to form conclusions and recommendations for the review. Synthesis methods

Where review authors pool the results of included studies, they should make some assessment that this pooling is appropriate, e. g. through tests of heterogeneity. If such a test is reported, then the analysis should be defined accordingly, e.g. by using a randomeffects model.

\section{Publication bias}

Assessment for publication bias should include graphical examination of the effect estimates of included studies against the precision of the study, e.g. in the form of a funnel plot.

Conflicts of interest

The conflicts of interest of the review authors should be made clear. However, conflicts of interest and sources of support in the included studies should also be examined. of the effects of the included studies, the authors undertook a fixed-effects meta-analysis. Effects were also summarised by subgroups, based on the type of cholinergic drug that was trialled, the age of participants and the duration of treatment. The reviewers conducted sensitivity analyses to assess the role of randomisation, assumptions made by the reviewers in respect of missing data, the risk of bias and the choice of meta-analytic model used.

\section{The results of the review}

As for the results, 14 studies involving 364 participants (study size ranged from $n=5$ to $n=60$ ) were included in qualitative and quantitative syntheses. All included studies were randomised and doubleblind, employing a parallel longitudinal design. Eight studies (Tarsy 1977; Jackson 1978, 1981; Lucius 1978; Kocher 1980; Gelenberg 1990; Caroff 2007; Ogunmefun 2009) employed a crossover design with two periods. Four studies (Tarsy 1977; Jackson 1978; Gelenberg 1990; Caroff 
2007) were undertaken for longer than 6 weeks; follow-up periods in the remaining studies ranged from 9 days and 6 weeks. Participants varied widely in demographic characteristics, but were mostly men in their 50s. All participants had clinically diagnosed tardive dyskinesia, or tardive dyskinesia identified using research diagnostic criteria laid down by Schooler and Kane (Schooler 1982). The majority of studies were conducted in hospital settings. Eight studies were conducted in the USA, and all studies were conducted in highincome countries, with the exception of one, which was carried out in Iran (Jahanian 2014).

The studies included for review were on deanol, lecithin, meclofenoxate hydrochloride, galantamine, rivastigmine and donepezil. All compared intervention with placebo. Scales employed in the included studies were the Abnormal Involuntary Movement Scale (AIMS) (Lane 1985), the Tardive Dyskinesia Rating Scale (TDRS) (Simpson 1979) and the Clinical Global Impressions scale (CGI) (Guy 1976), and most studies also commented on changes in mental state (without the use of scales) and adverse effects such as extra-pyramidal sideeffects (which were not described in detail). Forty-

\section{BOX 2 Methods of randomisation in intervention trials}

Randomised controlled trials usually randomise individual patients to one of a number of conditions, e.g. a treatment of interest and a placebo condition. In some instances, the randomised units (arms) may be aggregates of individuals, e.g. general practices or community mental health teams. Such trials are known as cluster randomised controlled trials.

\section{Simple randomisation}

Each patient sequentially entered into a randomised trial could be allocated to either the treatment or the control arm by the toss of a coin - here, each patient has an equal chance of either trial condition. Computers can also do this. Although simple randomisation should lead to similar numbers of patients in each arm if enough patients are randomised, in practice this method commonly leads to imbalance in size between groups, especially in trials involving a small number of participants.

\section{Permuted-block randomisation}

Permuted-block randomisation ensures balance in numbers of patients between treatment arms at certain points in the recruitment process. For example, with a block size of 10 , within each block of ten patients, five will be allocated to the intervention and five to the control condition, with random ordering of treatment allocations within each block. In non-blind trials, this method can introduce problems, e.g. if the block size is small.

\section{Stratified randomisation}

The above methods leave open the possibility of imbalance between the groups on important risk factors for the outcome (e.g. in the severity of the underlying illness). Stratified randomisation allows reasonable balance on such important factors between the arms of the trial.

\section{Dynamic catch-up}

This is a way of ensuring reasonable similarity in group size between the arms of a randomised trial. Once the difference between group sizes reaches a pre-specified level, it allows a group that is lagging behind another in size to 'catch up', by increasing the probability of allocation to that group.

\section{Minimisation}

This ensures a close balance between groups on several patient factors: in a sequence of allocations, the next patient's allocation probability is changed to minimise imbalance on pre-specified factors between the groups of the trial. nine studies were excluded, mostly because they were not randomised; other reasons for exclusion were that patients were not stabilised on a dose of antipsychotic by the time of trial start (Simpson 1977), no usable data were reported (Maršálek 1994) or, for cross-over trials, no data were reported at the end of the first phase (Chien 1978; Jus 1978; Penovich 1978; Perez-Cruet 1981; Domino 1985; Joe 1985; Nasrallah 1986; Lieberman 1988; Gelenberg 1990). For six of these excluded studies, authors were contacted, but no response was received. For a further two (Chien 1978; Maršálek 1994), no contact details for the authors were found. Two studies (Lucius 1978; Gelenberg 1990) described random allocation of participants in detail, but the others did not (Box 2 summarises possible methods of randomisation commonly applied in trials). Although all of the studies were described as double-blind, only three (Simpson 1977; Jackson 1979; Beckham 1983) described the blinding (masking) in detail, and none evaluated its effectiveness. Two studies (Beckham 1983; Jahanian 2014) reported outcome data as previously stated in a published protocol. All other studies did not report complete outcomes. All studies had small samples.

Three of the included studies were new to this update. These were trials of new cholinergic agents mainly used for the treatment of Alzheimer's disease: donepezil (Ogunmefun 2009), galantamine (Caroff 2007) rivastigmine (Jahanian 2014).

The number of participants included in the review was small, risking the reliability of its findings. No studies reported quality-of-life measures, limiting the ability of the reviewers to test this hypothesis. There was limited evidence for clinically important differences in deterioration of tardive dyskinesia symptoms between cholinergic drugs and placebo. Information on deterioration of mental state in people treated with cholinergic drugs was also of poor statistical quality. Adverse events were poorly examined and estimates imprecise. No statistical differences were noted between the proportion of participants leaving the studies in either arm. One study (George 1981) compared individuals randomised to placebo with those treated with $1 \mathrm{~g}$ or $2 \mathrm{~g}$ of deanol, finding greater improvements in the arm treated with the higher dose. However, the reviewers conclude that evidence on this remains uncertain, as this was a small study $(n=22)$ and there was limited available information on mental state, adverse events and the other outcomes of interest for the review.

\section{Conclusions}

The review was underpowered to answer the main question, which was 'Are cholinergic drugs clinically 
effective for tardive dyskinesia?'. The reviewers point out that the cholinergic action of older cholinergic drugs is unclear, and that newer cholinergics (e.g. rivastigmine) have not been fully assessed for tardive dyskinesia in randomised controlled trials. It is quite clear that the quality of evidence reviewed here is low, because of the small number of studies, small overall number of participants, selective and incomplete reporting of methods and results, and considerable uncertainty in the effect estimates, which might indicate either clinically important benefit or harm, of cholinergic drugs for tardive dyskinesia. The reviewers point out limitations of their review approach, particularly that they might have missed studies that should have been included, especially as these studies could well have been small in terms of sample size.

The findings of the review are consistent with the two previous Cochrane reviews on this question (Soares-Weiser 1997; Tammenmaa 2002). There is theoretical justification for the hypothesis that cholinergic drugs benefit tardive dyskinesia. However, cholinergic drugs are associated with adverse effects and, given that there is no evidence for their efficacy in tardive dyskinesia, it would be understandable if a patient with tardive dyskinesia would rather avoid these drugs. In the event of serious impairing tardive dyskinesia, the patient may wish to try a newer cholinergic, especially if it is prescribed in the context of a well-designed, properly reported clinical trial. There is insufficient evidence to recommend the use of cholinergic drugs to treat tardive dyskinesia in day-to-day clinical practice. The review authors point out that, although cross-over designs are useful in this area of study, where the number of eligible participants in the general population is small, they may well introduce bias because of carry-over effects. Larger sample sizes are needed. Researchers should also study a larger range of outcomes, including quality of life.

\section{References}

Beckham B (1983) Lecithin Therapy for Tardive Dyskinesia [dissertation]. North Texas State University.

Caroff SN, Walker P, Campbell C, et al (2007) Treatment of tardive dyskinesia with galantamine: a randomized controlled crossover trial. Journal of Clinical Psychiatry, 68: 410-5.

Chien C, Jung K, Ross-Townsend A (1978) Efficacies of agents related to GABA, dopamine, and acetylcholine in the treatment of tardive dyskinesia [proceedings]. Psychopharmacology Bulletin, 14: 20-22.

Domino EF, May WW, Demetriou S, et al (1985) Lack of clinically significant improvement of patients with tardive dyskinesia following phosphatidylcholine therapy. Biological Psychiatry, 20: 1189-96.

Gelder M, Gath D, Mayou R (1989) Oxford Textbook of Psychiatry. Oxford University Press.

Gelenberg AJ, Dorer DJ, Wojcik JD, et al (1990) A crossover study of lecithin treatment of tardive dyskinesia. Journal of Clinical Psychiatry, 51: 149-53.
George J, Pridmore S, Aldous D (1981) Double blind controlled trial of deanol in tardive dyskinesia. Australian and New Zealand Journal of Psychiatry, 15: 68-71.

Gerlach J, Casey DE (1988) Tardive dyskinesia. Acta Psychiatrica Scandinavica, 77: 369-78.

Guy W (1976) Clinical Global Impressions (CGI). In ECDEU Assessment Manual for Psychopharmacology (Revised DHEW Publication no. (ADM) 76-338): 217-22. National Institute of Mental Health.

Higgins JPT, Green S (eds) (2011) Cochrane Handbook for Systematic Reviews of Interventions: Version 5.1.0. Cochrane Collaboration.

Jackson I (1978) Cholinergic enhancement in tardive-dyskinesia. Current Therapeutic Research, Clinical and Experimental, 24: 725-33.

Jackson I, Nuttall E, Ibe I, et al (1979) Treatment of tardive dyskinesia with lecithin. American Journal of Psychiatry, 136: 1458-60.

Jackson IV, Davis LG, Cohen RK, et al (1981) Lecithin administration in tardive dyskinesia: clinical and biomedical correlates. Biological Psychiatry, 16: 85-90.

Jahanian AA, Rezaei 0, Fadai F, et al (2014) The effectiveness of rivastigmine in reducing tardive dyskinesia symptoms in patients with schizophrenia. Iranian Journal of Psychiatry \& Clinical Psychology, 20: 29-34.

Joe S, Suh K, Lee B (1985) Effect of lecithin on tardive dyskinesia. Korea University Medical Journal, 22: 197-206.

Jones PB, Barnes TRE, Davies L, et al (2006) Randomized controlled trial of the effect on quality of life of second- vs first-generation antipsychotic drugs in schizophrenia: Cost Utility of the Latest Antipsychotic Drugs in Schizophrenia Study (CUtLASS 1). Archives of General Psychiatry, 63: 1079-87.

Jus A, Villeneuve A, Gautier J, et al (1978) Deanol, lithium and placebo in the treatment of tardive dyskinesia. Neuropsychobiology, 4: 140-9.

Kane JM, Smith JM (1982) Tardive dyskinesia: prevalence and risk factors, 1959 to 1979. Archives of General Psychiatry, 39: 473-81.

Kocher R, Hobi V, Linder M, et al (1980) Therapy with dimethylaminoethanol (deanol) in late dyskinesias induced by neuroleptics. Schweizer Archiv für Neurologie und Psychiatrie, 126: 103-9.

Lane RD, Glazer WM, Hansen TE, et al (1985) Assessment of tardive dyskinesia using the Abnormal Involuntary Movement Scale. Journal of Nervous and Mental Disease, 173: 353-7.

Lieberman JA, Pollack S, Lesser M, et al (1988) Pharmacologic characterization of tardive dyskinesia. Journal of Clinical Psychopharmacology, 8: 254-60.

Lohr JB, Kuczenski R, Niculescu AB (2003) Oxidative mechanisms and tardive dyskinesia. CNS Drugs, 17: 47-62.

Lucius G (1978) Uber die therapeutische Wirksamkeit von Dimethylaminoaethanol bei neuroleptikainduzierten Späthyperkinesen [Dissertation]. Der Albert-Ludwigs-Universität Freiburg im Breisgau.

Maršálek M, Filip V, Prašková H, et al (1994) An open trial with 7-methoxytacrine in tardive dyskinesia. European Neuropsychopharmacology, 4: 369.

McEvoy JP, Lieberman JA, Stroup TS, et al (2006) Effectiveness of clozapine versus olanzapine, quetiapine, and risperidone in patients with chronic schizophrenia who did not respond to prior atypical antipsychotic treatment. American Journal of Psychiatry, 163: 600-10.

Miller DD, McEvoy JP, Davis SM, et al (2005) Clinical correlates of tardive dyskinesia in schizophrenia: baseline data from the CATIE schizophrenia trial. Schizophrenia Research, 80: 33-43.

Nasrallah HA, Dunner FJ, McCalley-Whitters M, et al (1986) Pharmacologic probes of neurotransmitter systems in tardive dyskinesia: implications for clinical management. Journal of Clinical Psychiatry, 47: 56-9.

Ogunmefun A, Hasnain M, Alam A, et al (2009) Effect of donepezil on tardive dyskinesia. Journal of Clinical Psychopharmacology, 29: 102-4.

Penovich P, Morgan M, Kerzner M (1978) Double-blind evaluation of deanol. JAMA, 239: 1997-8.

Perez-Cruet J, Menendez I, Alvarez-Ghersi J, et al (1981) Double-blind study of lecithin in the treatment of persistent tardive dyskinesia. Boletín de la Asociación Médica de Puerto Rico, 73: 531 
Schooler NR, Kane JM (1982) Research diagnoses for tardive dyskinesia. Archives in General Psychiatry, 39: 486-7.

Simpson GM, Voitashevsky A, Young MA, et al (1977) Deanol in the treatment of tardive dyskinesia. Psychopharmacology, 52: 257-61.

Simpson G, Lee J, Zoubok B, et al (1979) A rating scale for tardive dyskinesia. Psychopharmacology, 64: 171-9.

Soares-Weiser K, Mobsy C, Holliday E (1997) Anticholinergic medication for neuroleptic-induced tardive dyskinesia. Cochrane Database of Systematic Reviews, 2: CD000204 (doi: 10.1002/14651858.CD000204).

Solmi M, Pigato G, Kane JM, et al (2018) Clinical risk factors for the development of tardive dyskinesia. Journal of the Neurological Sciences, 389: 21-27
Stegmayer K, Walther S, van Harten P (2018) Tardive dyskinesia associated with atypical antipsychotics: prevalence, mechanisms and management strategies. CNS Drugs, 32: 135-47.

Tammenmaa I, McGrath J, Sailas EE, et al (2002) Cholinergic medication for neuroleptic-induced tardive dyskinesia. Cochrane Database of Systematic Reviews, 3: CD000207 (doi: 10.1002/14651858.CD000207).

Tammenmaa-Aho I, Asher R, Soares-Weiser K, et al (2018) Cholinergic medication for antipsychotic-induced tardive dyskinesia. Cochrane Database of Systematic Reviews, 3: CD000207 (doi: 10.1002/14651858. CD000207.pub2).

Tarsy D, Bralower M (1977) Deanol acetamidobenzoate treatment in choreiform movement disorders. Archives of Neurology, 34: 756-8. 